\title{
LC GENRE/FORM TERMS FOR RELIGIOUS MATERIAL PROJECT
}

LC's original timeline called for the Religion genre project to run from mid-2010 to mid2011. The end of April 2011 is the date for a first draft of proposed terms. There is still plenty of time to suggest new terms. There are currently, for example, people working on terms for Judaism, East Asian religions, and Eastern Orthodox Christianity.

Recently, Cameron Campbell, Barbara Kemmis and I had a conference call with Janis Young from LC regarding the next steps for the Religion genre/form project.A review of the terms from the December 9, 2010 Project Update resulted in an agreement to set aside all terms that were not considered by the project participants to constitute an appropriate as a genre term.

Janis also made the following suggestions:

1) For now, go to the broader level for many terms. For example, propose the term "Prayers" with a scope note indicating the term is to be used for Bedtime prayers, Pastoral prayers, etc. If LC decides later that more specific genre terms are needed, they will ask us to develop them. An exception to this would be a term where specificity is necessary; see, for example, the comments under "Didactic poetry, Arabic" on the Project wiki.

2) For now, cumulate but don't develop suggestions for music terms. They can later be referred to the Music genre/form project.

3) For now, cumulate but don't develop suggestions for literary terms. They can later be referred to the Literature genre/form project.

April is still the deadline for our first draft. Once that is circulated and we've accumulated comments, we will probably need a small group of volunteers to help develop subsequent drafts. Once we send LC a final draft, there will still be the need for further discussion. LC may have questions about some of the proposals or suggestions for revisions. The project may be completed by the end of 2011 .

We will try to add a short session on the Religion genre project to the program for ATLA's annual conference which will be held in Chicago June 8-11, 2011. You may be aware of other venues at which you can distribute drafts and solicit comments.

If you want access to the wiki but have forgotten your password or encounter difficulties accessing the website, please don't hesitate to email me. Thank you for your participation in this important project.

Submitted by Erica Treesh

Database Manager for Authority Control, ATLA Religion Database

American Theological Library Association

\section{SELECTED ARTICLES FROM CURRENT LIBRARY JOURNALS}

Bright, Alice, et al. "Minding Our Ps and Qs: An Adventure in Reclassification." Technical Services Quarterly 28, no. 1 (2011): 41-54. 